\title{
R7BP Complexes With RGS9-2 and RGS7 in the Striatum Differentially Control Motor Learning and Locomotor Responses to Cocaine
}

\author{
Garret R Anderson',3, Yan Cao',3, Steve Davidson², Hai V Truong ${ }^{2}$, Marco Pravetoni', Mark J Thomas', \\ Kevin Wickman', Glenn J Giesler Jr² and Kirill A Martemyanov*, I \\ 'Department of Pharmacology, University of Minnesota, Minneapolis, MN, USA; '² Department of Neuroscience, University of Minnesota, \\ Minneapolis, MN, USA
}

\begin{abstract}
In the striatum, signaling through $\mathrm{G}$ protein-coupled dopamine receptors mediates motor and reward behavior, and underlies the effects of addictive drugs. The extent of receptor responses is determined by RGS9-2/G $\beta 5$ complexes, a striatally enriched regulator that limits the lifetime of activated $G$ proteins. Recent studies suggest that the function of RGS9-2/G $\beta 5$ is controlled by the association with an additional subunit, R7BP, making elucidation of its contribution to striatal signaling essential for understanding molecular mechanisms of behaviors mediated by the striatum. In this study, we report that elimination of R7BP in mice results in motor coordination deficits and greater locomotor response to morphine administration, consistent with the essential role of R7BP in maintaining RGS9-2 expression in the striatum. However, in contrast to previously reported observations with RGS9-2 knockouts, mice lacking R7BP do not show higher sensitivity to locomotor-stimulating effects of cocaine. Using a striatum-specific knockdown approach, we show that the sensitivity of motor stimulation to cocaine is instead dependent on RGS7, whose complex formation with R7BP is dictated by RGS9-2 expression. These results indicate that dopamine signaling in the striatum is controlled by concerted interplay between two RGS proteins, RGS7 and RGS9-2, which are balanced by a common subunit, R7BP.

Neuropsychopharmacology (2010) 35, 1040-1050; doi:10.1038/npp.2009.212; published online 30 December 2009
\end{abstract}

Keywords: drugs of abuse; RGS proteins; basal ganglia; G protein signaling; motor control; dopamine receptors

\section{INTRODUCTION}

Dopamine signaling in the striatum has an essential role in controlling movement and reward processing (Groenewegen, 2003; Pierce and Kumaresan, 2006). Dopamine acts on two classes of G protein-coupled receptors (GPCR), which are differentially expressed in distinct populations of striatal neurons. Although $\mathrm{G}_{\text {olf-coupled }} \mathrm{D} 1$ receptors are mainly expressed on striatonigral ('direct') medium spiny neurons, $\mathrm{G}_{\mathrm{i} / \mathrm{o}}$-coupled $\mathrm{D} 2$ receptors are enriched in striatopallidal ('indirect') neurons (reviewed in Kreitzer, 2009). It is commonly accepted that balancing of signaling through these dopamine receptors is essential for the striatal control of movement and reward (Nicola et al, 2000; Kreitzer and Malenka, 2008). At a molecular level, the extent of dopamine action is controlled both presynaptically through

*Correspondence: Dr KA Martemyanov, Department of Pharmacology, University of Minnesota, 6-120 Jackson Hall, 32I Church Street S.E., Minneapolis, MN 55455, USA, Tel: +612 626 5309, Fax: +612 625 8408, E-mail: martemyanov@umn.edu

${ }^{3}$ These two authors contributed equally to this work.

Received 10 August 2009; revised 23 November 2009; accepted 30 November 2009 regulating the rate of the dopamine release and re-uptake, and postsynaptically by regulating the lifetime of receptor activity. Both of these mechanisms contribute essentially to normal dopamine signaling homeostasis (Gainetdinov et al, 2004) and their dysregulation has been implicated in a range of disorders including Parkinson's and Huntington's diseases, schizophrenia, and drug addiction (Hyman et al, 2006; DeLong and Wichmann, 2007).

Regulator of $G$ protein signaling (RGS) proteins are powerful negative regulators of GPCR responses that act to accelerate the inactivation of $\mathrm{G}$ protein $\alpha$ subunits, thereby controlling the duration of receptor signaling (Hollinger and Hepler, 2002; Burchett, 2005). A member of the RGS family, RGS9-2, is selectively enriched in the striatum in which it has been linked to the regulation of D2 receptor activity (Rahman et al, 2003; Cabrera-Vera et al, 2004; Kovoor et al, 2005). Mice lacking RGS9-2 develop motor coordination deficits (Blundell et al, 2008) and show enhanced locomotor responses to the administration of psychostimulants and opioids (Rahman et al, 2003; Zachariou et al, 2003; Kovoor et al, 2005). Furthermore, inactivation of RGS9-2 in mice (Kovoor et al, 2005) has been reported to exacerbate the development of 
haloperidol-induced tardive dyskinesia, whereas its overexpression in rats and primates (Gold et al, 2007) substantially reduced L-dopa-induced involuntary movements in Parkinsonian models. Together, these and other observations (see Traynor et al, 2009 for a comprehensive review) firmly establish RGS9-2 in the control of striatal dopamine signaling. However, details linking these behavioral observations to RGS9-2 function at the molecular level have been very limited.

RGS9-2 forms as a constitutive complex with the type $5 \mathrm{G}$ protein $\beta$ subunit $(\mathrm{G} \beta 5$ ) (reviewed in Sondek and Siderovski, 2001), and knockout of $\mathrm{G} \beta 5$ in mice leads to the elimination of detectable RGS9-2 (Chen et al, 2003). More recently, a third subunit in the complex was identified, the membrane anchor R7-binding protein (R7BP) (Martemyanov et al, 2005; Drenan et al, 2005), which further controls the proteolytic stability of RGS9-2/G $\beta 5$ complex and targets it to postsynaptic sites (Anderson et al, 2007b). In addition to RGS9-2, both G $\beta 5$ and R7BP in the striatum form complexes with another equally abundant RGS9-2-like protein, RGS7 (Anderson et al, 2009a). RGS9-2/G $\beta 5$ and RGS7/G $\beta 5$ are coupled to R7BP in a dynamic manner and changes in RGS9-2 levels (eg, as observed in RGS9 knockout mice) dramatically affect $\mathrm{RGS7/G} \beta 5$ localization. The behavioral implications of this complexity in subunit organization of RGS complexes are unknown, however, largely because of the lack of functional understanding of the role of R7BP and RGS7 in the basal ganglia.

In this study, we report that elimination of R7BP results in motor coordination deficits and increase in sensitivity to locomotor-stimulating effects of morphine, consistent with the essential role of R7BP in maintaining RGS9-2 expression in the striatum. However, in contrast to previously reported observations with RGS9-2 knockouts, the motor responses of mice lacking R7BP to acute or chronic administration of cocaine were indistinguishable from their wild-type littermates. Using a striatum-specific knockdown approach, we show that the sensitivity of locomotor responses to cocaine is instead dependent on the expression of RGS7. These results suggest that dopamine signaling in the striatum is controlled by concerted interplay between two RGS proteins, RGS7 and RGS9-2, and balanced by a common subunit, R7BP.

\section{MATERIALS AND METHODS}

\section{Animals}

All studies were carried out in accordance with the National Institute of Health guidelines and were granted formal approval by the Institutional Animal Care and Use Committee of the University of Minnesota. The generation of R7BP knockouts (R7BP ${ }^{-1-}$ ) was described earlier (Anderson et al, 2007b). The line was out-bred onto the C57BL/6J background for five generations. All animals used for comparison of $\mathrm{R} \mathrm{BP}^{-1-}$ and $\mathrm{R}^{-1 \mathrm{BP}^{+/+}}$genotypes were littermates derived from heterozygous breeding pairs. Viral rescue studies were carried out with $\mathrm{R} \mathrm{BP}^{-1-}$ mice derived from homozygous $\mathrm{R}^{\mathrm{B}} \mathrm{BP}^{-1-}$ breeding pairs. Striatal knockdown studies for RGS7 were carried out with C57BL/6J mice purchased from Charles River and bred in house. Mice were housed in groups on a $12 \mathrm{~h}$ light-dark cycle with food and water available ad libitum. Only males 2-4 month of age were used.

\section{Accelerating Rotarod Test}

Motor coordination was measured by performance on an accelerating rotarod device equipped with drums sized for mice (IITC Life Science). Littermate mice were habituated in the test room for an hour before the testing. Three trials were performed per day over 3 days for a total of nine trials for each animal. After placing a mouse on the rod, it was accelerated from 4 to 27 r.p.m. in $5 \mathrm{~min}$. The endurance of mice on the rotarod was measured by time to fall to the floor of the apparatus, or to turn around one full revolution while hanging onto the drum.

\section{Open Field Locomotor Activity Test}

Littermate mice were habituated to the test room environment for $1 \mathrm{~h}$ before testing. Activity studies were performed in Plexiglas chamber (ENV-515; $170 \mathrm{~W} \times 170 \mathrm{~L} \times 120 \mathrm{H}$; Med Associates, St Albans, VT) as described earlier (Pravetoni and Wickman, 2008). Briefly, a mouse was placed in the center of the open field arena and allowed to freely move for 60 or $180 \mathrm{~min}$ while being tracked by an automated tracking system consisting of three 16 beam infrared arrays, the data from which were analyzed by Open Field Activity software (Med Associates). Infrared beam break data were collected in $5 \mathrm{~min}$ bins and used to extract ambulatory activity (crossing four beams within $500 \mathrm{~ms}$ ), and distance traveled. Beam break activity in the absence of consecutive crossing of beams was defined as steretotypic movements. Thigmotaxis (wall hugging) for each subject was determined by dividing the distance traveled in the 7.5$\mathrm{cm}$ wide perimeter of the environment by the total distance traveled during the 180 -min session. The number of entries in the central area of the open field (total area minus outer perimeter) was also measured. In studies measuring the dose-dependence effects of the drugs on the locomotor activity, mice were injected subcutaneously with saline or increasing doses of morphine or cocaine $(1,5,10$, and $20 \mathrm{mg} / \mathrm{kg}$ ) freshly dissolved in saline with a 3-day interval between the injections. For cocaine sensitization studies, mice were injected intraperitonealy daily: first with saline for 3 days, followed by $10 \mathrm{mg} / \mathrm{kg}$ cocaine for 5 consecutive days. Animal activity was measured immediately on the injection and recorded for 60 (cocaine) or 180 (morphine) minutes.

\section{RNA Interference and Generation of Recombinant Lentiviruses}

RGS7 expression was downregulated by short interfering RNA duplexes similarly as described before for R7BP (Anderson et al, 2007a). Target regions in RGS7 were identified and miR RNAi sequences were designed by BLOCK-iT RNAi Designer Program (Invitrogen). RNAi molecules were generated by targeting the 427-447 coding region of the RGS7 gene (RNAi 427, sequence $5^{\prime}$-CTA GAGCTAGCAGATTATGAA- $3^{\prime}$ ). Synthetic double-stranded oligonucleotides corresponding to the designed RNAi was cloned into the pcDNA6.2GW/EmGFP vector in the middle 
of the micro RNA 155 (miR155) sequence supplied as a part of the BLOCK-iT Lentiviral Pol II miR RNAi expression system kit (Invitrogen). In the pcDNA6.2GW/EmGFP vector, the chimeric miR155-RGS7 sequence is located under the control of the cytomegalovirus promoter cocistronically with EmGFP. On processing in the cells by the endogenous machinery, the construct is used to produce anti-RGS7 RNA duplex (miRNA- $\alpha$ RGS7). The control construct (miRNA-CTR) was created by cloning a scrambled sequence $5^{\prime}$-AAATGTACTGCGCGTGGAGAC- $3^{\prime}$ into the miR155 environment identically as described for miRNA- $\alpha$ RGS7. The expression cassette was transferred to the lentiviral shuttle vector pLenti6/V5-DEST vector (Invitrogen) by Gateway recombination following the kit instructions.

For the generation of infectious lentiviral particles, pLenti6/V5-DEST vectors containing miRNA- $\alpha$ RGS7 or miRNA-CTR cassettes were co-transfected with the packaging plasmid mixture (kindly provided by Professor Didier Trono of the Universite de Geneve): pRSV-Rev, pMDLg/ pRRE, and pMD2.G into 293T cells using Lipofectamine 2000 (Invitrogen). Fifteen T75 flasks were used to produce each batch of lentivirus. Virus-containing media was collected $60-65 \mathrm{~h}$ after transfection, filtered through a $0.45-\mu \mathrm{m}$ filter (Millipore), and viral particles were enriched by passing through a $500 \mathrm{kDa}$ MidiKros hollow fiber column (Spectrum Labs) until the retentate volume was reduced to approximately $20-25 \mathrm{ml}$ of media. Viral particles were further concentrated as described (Coleman et al, 2003) with some modifications. Virus-containing supernatants were carefully loaded on 60\% OptiPrep (Sigma) cushion $(150-200 \mu \mathrm{l})$ in $30-\mathrm{ml}$ conical-bottomed polyallomer centrifuge tubes (Beckman) and centrifuged at $20000 \times \mathrm{g}$ for $2.5 \mathrm{~h}$ at $4{ }^{\circ} \mathrm{C}$ using a swinging bucket rotor SW-28 (Beckman). The medium just above the media/OptiPrep interface was carefully removed and discarded. The residual medium-containing OptiPrep and viruses $(\sim 200 \mu \mathrm{l}$ each tube) were resuspended by gentle pipetting, aliquoted, and stored at $-80^{\circ} \mathrm{C}$ until use.

Infectious titers of viruses were determined by Lenti-X qRT-PCR Titration Kit (Clontech). Our preparations of concentrated lentiviral stocks consistently yielded titers of $1-3 \times 10^{12}$ viral copies $/ \mathrm{ml}$. The titers of all viral stocks were equalized by adjusting the concentration of viral particles to $1 \times 10^{12} / \mathrm{ml}$.

\section{Cell Culture and Gene Transfer}

$293 \mathrm{~T}$ cells were obtained from ATCC and cultured at $37^{\circ} \mathrm{C}$ and $5 \% \mathrm{CO}_{2}$ in DMEM supplemented with antibiotics, $10 \%$ fetal bovine serum, $1 \mathrm{mM}$ sodium pyruvate, and $1 \mathrm{X}$ nonessential amino acids (Invitrogen). Cells were transfected at $\sim 70 \%$ confluency, using Lipofectamine 2000 (Invitrogen) according to the manufacturer's protocol. The ratio of Lipofectamine to DNA used was $4 \mu \mathrm{l}: 2.5 \mu \mathrm{g} / 4 \mathrm{~cm}^{2}$ of cell surface. The cells were grown for $24-48 \mathrm{~h}$ post-transfection, washed with PBS, lysed in SDS sample buffer $(62 \mathrm{mM}$ Tris, $10 \%$ glycerol, $2 \%$ SDS, $5 \% \quad \beta$-mercaptoethanol) and analyzed by $4-20 \%$ PAGE (Cambrex).

Primary cultures of striatal neurons were essentially prepared as described earlier (Anderson et al, 2007a). The cultures were transduced by lentiviral constructs on day 4 in vitro by adding specified amounts of high-titer lentiviral stocks and the media was substituted on day 7. On day 14, cells were washed with PBS, and lysed in SDS sample buffer.

\section{Preparation of Striatal Tissue Extracts, Immunoprecipitation, and Western Blotting}

The whole brain from 25-day-old C57/BL6 mice were quickly removed and placed in an ice-cold slicing solution containing $85 \mathrm{mM} \mathrm{NaCl}, 2.5 \mathrm{mM} \mathrm{KCl}, 4 \mathrm{mM} \mathrm{MgCl}_{2}, 1 \mathrm{mM}$ $\mathrm{NaH}_{2} \mathrm{PO}_{4}, 25 \mathrm{mM} \mathrm{NaHCO}, 25 \mathrm{mM}$ glucose, and $75 \mathrm{mM}$ sucrose (all from Sigma, St Louis, MO) $\mathrm{pH}=7.4$. Coronal slices were prepared $300 \mu \mathrm{m}$ in thickness using a Vibratome 3000 Plus sectioning system (Vibratome, St Louis, MO). Striatal tissue was then isolated corresponding to viral type injection and directly frozen in liquid $\mathrm{N}_{2}$. Cellular lysates were prepared by homogenizing the isolated striatal tissue by sonication in immunoprecipitation (IP) buffer $(1 \times \mathrm{PBS}$, $150 \mathrm{mM} \mathrm{NaCl}, 1 \%$ Triton X-100, Complete (Roche) protease inhibitors) followed by 15 -min centrifugation at $14000 \times g$. The resulting extract was used for protein concentration determination by BCA assay (Pierce, Rockford, IL). IP of R7BP was performed using $2 \mu \mathrm{g}$ of sheep anti-R7BP NT antibody, $10 \mu$ l of protein G beads (GE Healthcare, Little Chalfont, UK), added to the extracts. The mixtures were incubated for $1 \mathrm{~h}$, washed three times with IP buffer, and proteins bound to the beads were eluted with SDS sample buffer. Samples were resolved on SDS-PAGE gel, transferred onto PVDF membrane, and subjected to western blot analysis using HRP-conjugated secondary antibodies and ECL West Pico (Pierce) detection system.

Analysis was performed using sheep anti-RGS9-2 CT (Martemyanov et al, 2005), rabbit anti-RGS7 (7RC1), rabbit anti-R7BP antibodies (generously provided by William Simonds, National Institute of Diabetes and Digestive and Kidney Diseases, National Institutes of Health), and mouse monoclonal anti- $\beta$-actin (clone AC-15) antibodies purchased from Sigma.

\section{Immunohistochemistry}

Immunohistochemical detection of R7BP protein in striatal sections was performed after transcardiac perfusion of mice with $4 \%$ paraformaldehyde in $0.1 \mathrm{M}$ sodium phosphate buffer $(\mathrm{pH}=7.4)$. Brains were cryoprotected with $30 \%$ sucrose in PBS for 2 days at $4{ }^{\circ} \mathrm{C}$, and embedded in TissueTek OCT compound (Sakura Finetek). Embedded brains were frozen on dry ice, and 40-m frozen sections were obtained by cryostat (Leica). Sections were blocked in PT1 (PBS with $0.1 \%$ Triton X-100 and $10 \%$ goat serum) for $1 \mathrm{~h}$ and incubated with $1: 500$ dilution of primary rabbit antiR7BP (TRS) antibody (generously provided by William Simonds, National Institute of Diabetes and Digestive and Kidney Diseases, National Institutes of Health) in PT2 (PBS with $0.1 \%$ Triton X-100 and $2 \%$ goat serum) for $1 \mathrm{~h}$, washed four times for $5 \mathrm{~min}$ in PT2, and incubated with fluorophore-conjugated secondary antibodies (goat anti-rabbit Alexa 546, 1:1000) in PT2 for $1 \mathrm{~h}$. Sections were washed three times for $5 \mathrm{~min}$ in PT2 and mounted in Mounting Medium (Pierce). Images were taken by an Olympus Fluoview 1000 confocal microscope. 


\section{Stereotaxic Injections}

The development of the method for the regional delivery of substances to the brains of embryonic and neonatal mice is described in detail in a separate publication (Davidson et al, 2010). Briefly, 5-day-old mice were anaesthetized with iceinduced hypothermia, and concentrated lentivirus at a titer of $1 \times 10^{12}$ copies $/ \mathrm{ml}$ was bilaterally injected into the striatum at four depth locations per hemisphere with the tip of the microinjection pipette advanced to the deepest (ventral) position for the first injection; additional injections were made every $0.3 \mathrm{~mm}$ while withdrawing the injection needle. The coordinates for each bilateral injection from Bregma were: anterior $0.35 \mathrm{~mm}$, mediolateral $1.75 \mathrm{~mm}$, at dorsoventral depths of 3.1, 2.8, 2.5, and $2.2 \mathrm{~mm}$. For animals used for behavior analysis, $90 \mathrm{nl}$ of virus was injected at each depth point and the same virus was delivered bilaterally. For verification of protein expression or knockdown, $600 \mathrm{nl}$ of virus was used at each depth point and each animal received both control and experimental viruses on different sides. We used an electrode manipulator stand with a three-axis manipulator and an attached microinjection unit (Model 1449 Electrode Manipulator Stand, Model 1460-61 Electrode Manipulator, and Model 5000 Microinjection Unit; David Kopf Instruments, Tejunga, CA). A 5- $\mu$ l manual syringe (Model 95, Hamilton, Reno, NV) was fitted to the Microinjection Unit. Pipette tips were pulled from thin walled borosilicate glass capillary tubes (with filament) with an outer diameter of $1.0 \mathrm{~mm}$ and inner diameter of $0.76 \mathrm{~mm}$. Pipettes were backfilled with light mineral oil and then forward filled with concentrated lentiviruses or $4 \%$ Fluorogold (Fluorochrome, LLC, Denver, $\mathrm{CO})$ for visualization of injection site. All injections were carried out with the aid of a dissecting microscope. Anatomical borders in the perinatal mouse brain were determined with the assistance of the Atlas of the Developing Mouse Brain (Paxinos et al, 2007).

\section{Statistical Analysis}

Data were analyzed by statistics suite of SigmaPlot v. 11 software package. Two-way analysis of variance (ANOVA) was conducted on all of the experimental data using genotype and session number as grouping factors. Dunnet's and Tukey's post hoc tests were used for individual pairwise comparisons. Biochemical data were evaluated by Student's $t$-test. All data are reported as means with SEM values.

\section{RESULTS}

\section{Knockout of R7BP does not Alter Basal Behavioral Responses of Mice}

Mice with constitutive disruption of the R7BP gene $\left(\mathrm{R}^{\mathrm{B}} \mathrm{BP}^{-1-}\right)$ were born in accordance to normal Mendelian inheritance patterns, had overall appearance indistinguishable from their wild-type littermates, were fertile, and showed normal development. By age 2-4 months, adult $\mathrm{R} \mathrm{BP} \mathrm{B}^{-1-}$ mice reach normal body weights $(27.21 \pm 0.96 \mathrm{~g}$ male and $21.56 \pm 0.62 \mathrm{~g}$ female) compared with their wild-type R7BP ${ }^{+/+}$littermates $(27.12 \pm 0.96 \mathrm{~g}$ male and $21.54 \pm 0.47 \mathrm{~g}$ female $)$. Analysis of $\mathrm{R} \mathrm{BP}^{-1-}$ behavior in the open field test revealed normal baseline ambulatory activities both in terms of total distance traveled during the assay (Figure 1b) and average movement velocities (Figure 1c). As compared with their wild-type littermates, $\mathrm{R}_{\mathrm{BP}}{ }^{-1-}$ mice showed the same level of heightened exploratory behavior when introduced into the novel environment of the chamber. Both genotypes also showed indistinguishable habituation behavior, reducing their locomotor activities five to sixfold during the first $2 \mathrm{~h}$ (Figure 1a). Similarly, R7BP ${ }^{-1-}$ mice were indistinguishable from their wild-type littermates for their repetitive (Figure $1 \mathrm{~d}$ and e) and anxiety-related (Figure $1 \mathrm{f}$ and g) behaviors. In summary, these experiments show overall preservation of basal behavioral responses in $\mathrm{R} \mathrm{BP}^{-1-}$ mice.
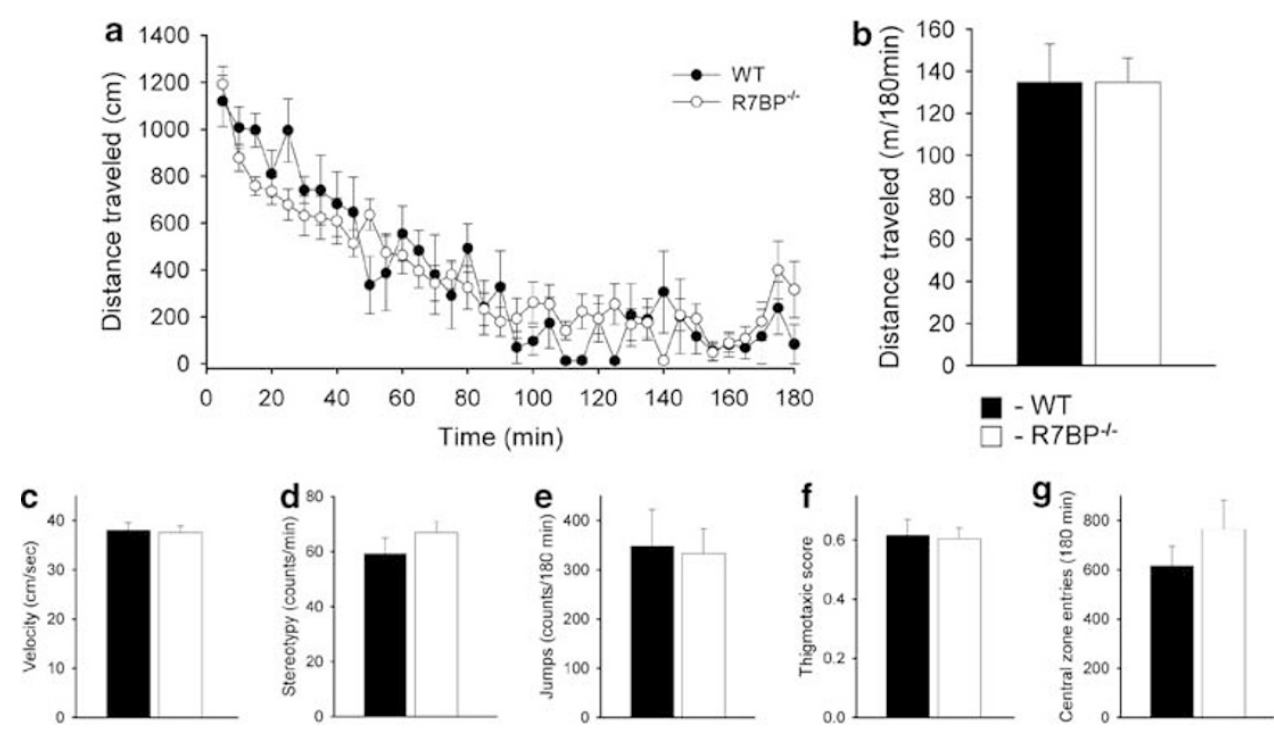

Figure I Baseline behavioral characteristics are unaltered on the genetic elimination of R7BP. R7BP knockout $\left(\mathrm{R}^{\mathrm{B}} \mathrm{BP}^{-1-}\right)$ mice $(n=9)$ and wild-type $\left(\mathrm{R} 7 \mathrm{BP}^{+1+}, \mathrm{WT}\right)$ litter mates $(n=5)$ were placed in locomotor open field test chamber for $3 \mathrm{~h}$ and analyzed for the behavioral traits. (a) Distance traveled per 5-min interval was plotted over the 3-h evaluation period, or used to calculate cumulative total distance traveled (b). Quantification and one-way ANOVA reveals that travel velocity (c), stereotypy counts (d), vertical jumps (e), thigmotaxis ( $\mathrm{f}$ ), and central zone entries (g) between R7BP ${ }^{-1-}$ and R7BP ${ }^{+/} /$ littermates were all statistically insignificant. 


\section{Loss of R7BP in the Striatum Leads to Motor Coordination Deficits}

Consistent with the severe reduction in the levels of RGS9-2 in the striatum on the loss of R7BP (Anderson et al, 2007b), evaluation of $\mathrm{R}^{\mathrm{B}} \mathrm{BP}^{-1-}$ mice in the accelerated rotarod test revealed their substantial deficiencies in gross motor coordination (Figure 2). Indeed, during 3 days of training, $\mathrm{R} \mathrm{BP}{ }^{-1-}$ mice showed consistently lower performance reflected by the shorter durations of balancing on the rotating drum (Figure 2a). Analysis of the data by two-way ANOVA indicated statistically significant effects of genotype (Figure 2b; $\mathrm{F}_{(1,180)}=23.955, p<0.001$ ) and trial number (not shown; $\mathrm{F}_{(8,180)}=20.601, p<0.001$ ). Post hoc pairwise comparison of animals grouped by trial day (Figure 2c) showed that while prominent on later trials, the genotype differences in animal performance on initial trials was absent, suggesting that slower rates of motor learning might underlie deficiencies of $\mathrm{R} \mathrm{BP}^{-l-}$ mice in the rotarod test.

Similar motor coordination deficits are also observed on elimination of R7BP's binding partner, RGS9-2 (Blundell et al, 2008), which is selectively enriched in the striatum (reviewed by Traynor et al, 2009). As R7BP was reported to be broadly expressed in the central nervous system, including regions outside of the striatum (Martemyanov et al, 2005; Grabowska et al, 2008) that are also implicated in controlling motor coordination (eg, cerebellum), we next addressed the specific contribution of striatal R7BP to the observed phenotype. This was achieved by selective expression of R7BP in the dorsal striatum of $\mathrm{R}^{\mathrm{B}} \mathrm{BP}^{-1-}$ mice by lentiviral gene transfer in neonatal mice. Before proceeding with the lentiviral manipulations in multiple mice to be used for behavioral experiment, control experiments evaluating the efficiency of the approach have been conducted with a few animals. After confirmation of selective targeting of the striatal regions in neonatal mice by Fluorogold injections (Supplementary Figure S1), test $\mathrm{R} 7 \mathrm{BP}^{-9-}$ mice received unilateral injections of lentiviral particles driving the expression of R7BP into the dorsal striatum (Figure 3a). The contra-lateral sides of the same animals were injected with the control virus expressing the unrelated $L a c Z$ gene. When mice reached maturity, their striatal regions within the injection plane were collected and analyzed for the expression of proteins of interest. Data presented in Figure $3 \mathrm{~b}$ revealed significant restoration of $\mathrm{R} 7 \mathrm{BP}$ expression in $\mathrm{R} \mathrm{BP}^{-1-}$ mice driven by lentiviral gene transfer. Consistent with earlier findings (Anderson et al, 2007a, b), lentiviral expression of R7BP resulted in the selective upregulation of the RGS9-2, but not RGS7 levels presumably because of the increased proteolytic protection of the complex. Furthermore, lentiviral expressed R7BP coprecipitated with RGS9-2, and to a lesser extent with RGS7, an indication of its functional status.

Having confirmed the effectiveness of the manipulations, for subsequent behavioral studies neonatal mice were injected bilaterally (Figure 3a). The mice were tested 2 months after the injection at which point the expression of R7BP in the striatum was verified immunohistochemically (Figure 3c). Data presented in Figure 3d and e illustrates that selective restoration of R7BP expression in the striatum leads to a substantial improvement of mouse performance in the rotarod test. Two-way ANOVA analysis (trial by virus) revealed a significant increase in fall latencies on R7BP restoration into the striatum $\left(\mathrm{F}_{(1,125)}=16.41\right.$, $p<0.001)$, and a significant effect of trial $\left(\mathrm{F}_{(8,125)}=9.62\right.$, $p<0.001)$ These data suggest that the motor coordination deficits observed in R7BP knockout mice are likely explained by the de-regulation of RGS9-2 function in the striatum.

\section{Elimination of R7BP does not Alter Locomotor Responses to Cocaine Administration}

In addition to motor coordination deficits, mice lacking RGS9-2 have been reported to develop hypersensitivity to the administration of drugs that increase synaptic dopamine concentration such as amphetamine, cocaine, and morphine (Rahman et al, 2003; Zachariou et al, 2003; Kovoor et al, 2005). We have, therefore, evaluated how elimination of R7BP affects locomotor responses of mice to psychostimulant administration. Both $\mathrm{R} \mathrm{BP}^{-1-}$ and $\mathrm{R}^{-1 \mathrm{BP}^{+/+}}$ littermates showed progressively greater responses to increasing doses of cocaine (Figure 4a; $\mathrm{F}_{(4,80)}=$ $23.128, p<0.001)$. However, no statistically significant differences in locomotor activities were observed between genotypes at any concentration of the tested drug (two-way
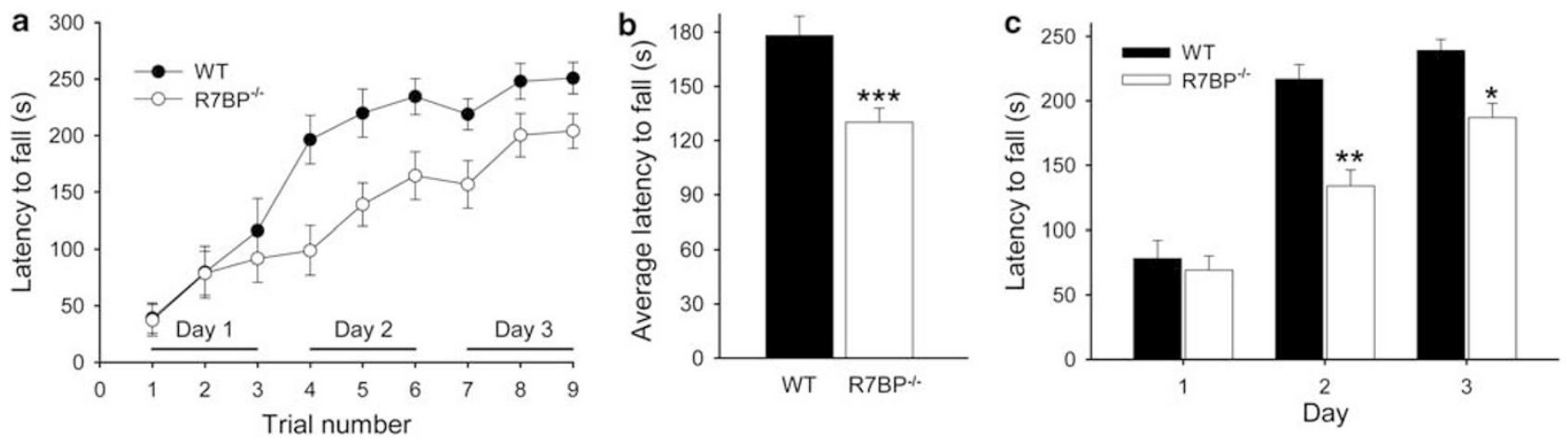

Figure $2 \mathrm{R} 7 \mathrm{BP}^{-1-}$ mice show deficits in motor coordination. (a) R7BP ${ }^{-1-}$ mice $(n=14)$ and $\mathrm{WT}(n=8)$ were analyzed using an accelerated rotarod test over the course of three trials/day, for 3 days totaling nine trials. A two-way ANOVA (trial by genotype) revealed a significant effect of genotype $\left(F_{(I, 180)}=23.96, p<0.00 \mathrm{I}\right)$, and a significant effect of trial $\left(F_{(8,180)}=20.60 \mathrm{I}, p<0.00 \mathrm{I}\right)$. (b) Calculated mean latencies to fall off the rotarod across all trials were $178 \pm 8\left(\mathrm{R} 7 \mathrm{BP}^{+1+}\right)$ and $130 \pm 6\left(\mathrm{R}_{\mathrm{BP}}{ }^{-1-}\right)$. (c) The mean latency to fall off the rotarod for the three trials for each day revealed a significant difference between genotypes on days $2(p<0.0 \mathrm{I})$ and $3(p<0.05)$. 

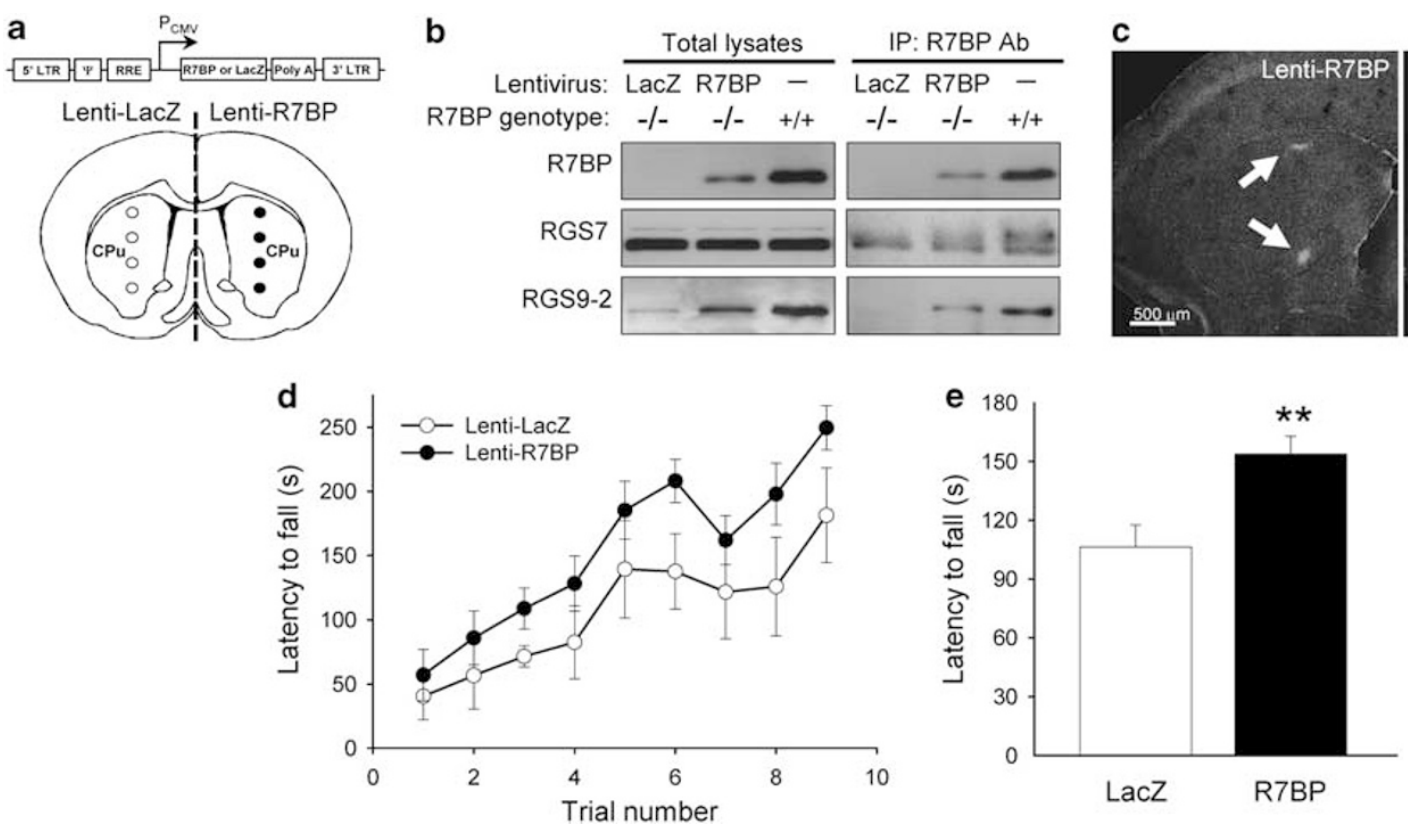

Figure 3 Lentiviral expression of R7BP in dorsal striatum improves the performance of R7BP ${ }^{-1-}$ in rotarod task. (a) Strategy for lentivirus-mediated protein expression in vivo. Shown schematic illustrates location of lentiviral construct (R7BP or LacZ) unilateral injection sites at four depth points (3. I, 2.8, 2.5, and $2.2 \mathrm{~mm}$ ) of 5-day-old R7BP ${ }^{-1-}$ mice. Each side of the brain received four 600-nl injections with either R7BP or control LacZ lentivirus within the striatum. (b) Verification of lentiviral-mediated R7BP expression in adult R7BP ${ }^{-1-}$ mice. Left, at postnatal day 25, brains were sectioned at 300- $\mu$ m thickness, and LacZ/R7BP lentivirus injected striatum was isolated. Tissue sections were solubilized with $1 \%$ Triton X-I 00 containing buffer, lysates containing I $0 \mu \mathrm{g}$ of total protein were separated on 12.5\% PAGE, and subjected to western blotting with the indicated antibodies. Shown is maximal expression of R7BP detected within the isolated sections. Maximal R7BP-mediated lentiviral expression in R7BP ${ }^{-1-}$ mice were estimated to be $\sim 30 \%$ of the corresponding levels in R7BP ${ }^{++}$mice using quantitative densitometry analysis with Imagej software package. Right, western blot analysis of proteins immunoprecipitated from the total lysates by $2 \mu \mathrm{g}$ of anti-R7BP antibodies. (c) Verification of R7BP expression around injection sites by immunostaining with anti-R7BP antibodies. R7BP ${ }^{-1-}$ mice were injected with $90 \mathrm{nl}$ of R7BP expressing lentivirus at each injection site, and killed at I0 weeks. In total, I-2 injection sites in a $40-\mu \mathrm{m}$ brain slice were typically observed in one plane, with two injection sites shown. (d) Performance of R7BP ${ }^{-1-}$ mice bilaterally injected with either R7BP $(n=9)$ or Lac $Z(n=5)$ expressing lentiviruses $(90 \mathrm{nl}$ per injections site) in rotarod test. ANOVA analysis revealed a significant increase in fall latencies on $R 7 B P$ restoration into the striatum $\left(F_{(I, 125)}=16.41, p<0.00 I\right)$ as well as a significant effect of trial $\left(F_{(8,125)}=9.62, p<0.00 I\right)(e)$. Mean latency to fall off the rotarod across all trials. Values are: $106 \pm$ I I s for LacZ injected mice and $153 \pm 9 \mathrm{~s}$ for mice injected with R7BP expressing virus.
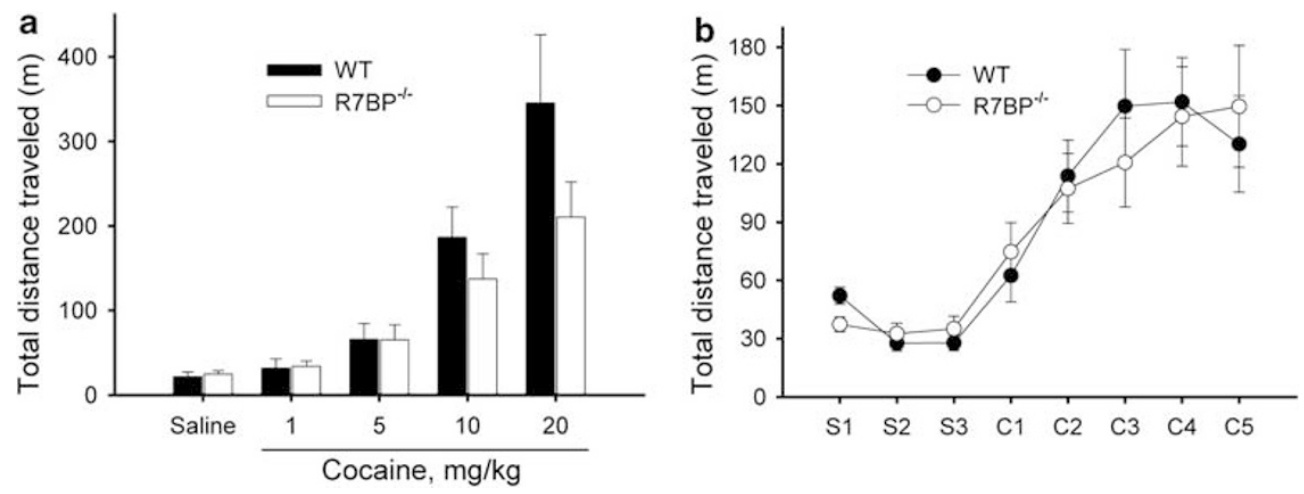

Figure 4 Locomotor stimulatory effects of cocaine administration are unaltered in $\mathrm{R}^{\mathrm{B}} \mathrm{BP}^{-1-}$ mice. (a) Locomotor activities of R7BP ${ }^{-1-}(n=12)$ and their wild-type litermates $(n=6)$ in response to increasing dozes of cocaine administration. Mice were injected with saline or cocaine $(1,5,10$, and $20 \mathrm{mg} / \mathrm{kg})$ and their locomotor activity was measured for I h. A two-way ANOVA (dose by genotype) revealed a significant effect of dose $\left(F_{(4,80)}=23.128, p<0.00 \mathrm{I}\right)$ but no effect of genotype $\left(F_{(I, 80)}=3.248, p=0.075\right)$. (b) Locomotor sensitization to repeated cocaine administration. Mice of both genotypes $(n=18$ for $\mathrm{R} \mathrm{BP}^{-1-}$ and $\mathrm{n}=1 \mid \mathrm{for} \mathrm{R}_{\mathrm{BPP}}^{+/+}$) were given daily injections of saline for 3 days (SI-3), followed by 5 daily injections of $10 \mathrm{mg} / \mathrm{kg} \mathrm{cocaine}(\mathrm{Cl}-\mathrm{C} 5)$. A twoway ANOVA (day by genotype) revealed a significant effect of trial number $\left(F_{(7,211)}=13.38, p<0.00 \mathrm{I}\right)$ as both groups showed higher locomotor activity with each additional cocaine injection (ie, sensitization). However, there was no statistical difference between genotypes $\left(F_{(1.211)}=0.30, p=0.86\right)$.

ANOVA: $\left.\mathrm{F}_{(1,80)}=3.248, p=0.075\right)$. Similarly, repetitive administration of the same cocaine concentration caused pronounced sensitization of mice to the drug, yet no differences in the extent of the responses were observed
(Figure 4b). Two-way ANOVA revealed that a significant effect of trial number $\left(\mathrm{F}_{(7,211)}=13.382, p<0.001\right)$ with no effect of genotype $\left(\mathrm{F}_{(1,211)}=0.3023, p=0.858\right)$. As morphine, in addition to increasing dopamine levels through 


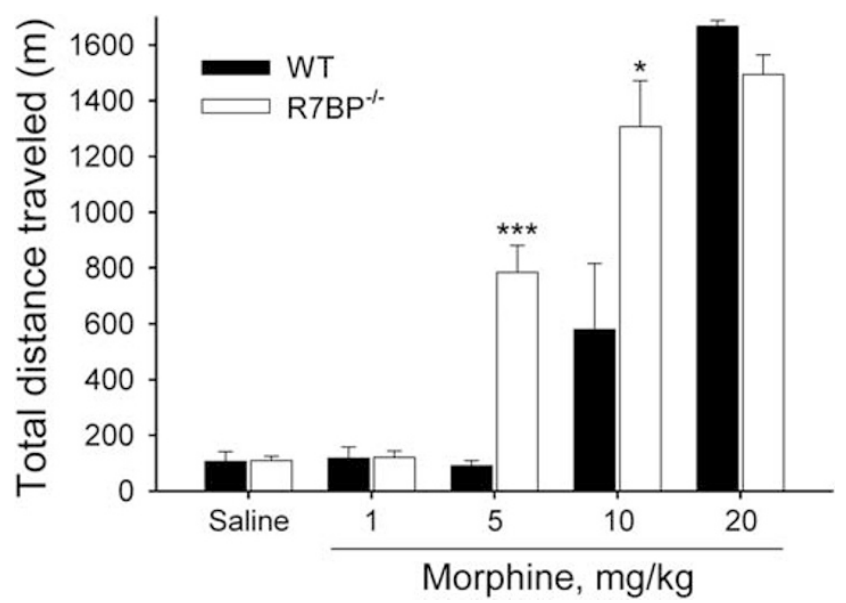

Figure 5 Increased sensitivity of $\mathrm{R} 7 \mathrm{BP}^{-1-}$ mice to locomotor-stimulating effects of morphine. Mice of both genotypes ( $n=9$ for R7BP ${ }^{-1-}$ and $n=5$ for $\mathrm{R}_{7 \mathrm{BP}^{+/+}}$) were injected with increasing doses of morphine $(\mathrm{I}, 5,10$, and $20 \mathrm{mg} / \mathrm{kg}$ ) and their locomotor activities were measured for $3 \mathrm{~h}$ after the administration. Total distance traveled in locomotor chambers was plotted as bar graphs. A two-way ANOVA (dose $\times$ genotype) revealed both a significant effect of dose $\left(F_{(4,60)}=71.99, p<0.00 I\right)$, and a significant effect of genotype $\left(F_{(1,60)}=14.37, p<0.001\right)$.

presynaptic mechanisms, can also affect signaling in striatal neurons directly by acting at $\mu$-opioid receptors (reviewed in Nestler, 2005), we have additionally compared locomotor responses of mice with morphine (Figure 5). $\mathrm{R} \mathrm{BP}{ }^{-1-}$ mice robustly responded to the administration of $5 \mathrm{mg} / \mathrm{kg}$ of morphine, a dose that failed to elicit a response in wild-type littermates. A significant difference in sensitivity remained apparent at the higher dose of $10 \mathrm{mg} / \mathrm{kg}$, while no differences was observed at the maximal concentration used $(20 \mathrm{mg} / \mathrm{kg})$. These data suggest that like RGS9-2deficient mice, $\mathrm{R} \mathrm{BP}{ }^{-1-}$ mice show increased sensitivity to the administration of morphine, however, unlike RGS9 ${ }^{-1-}$ mice, $\mathrm{R} \mathrm{BP}{ }^{-1-}$ mice do not have altered responses to the locomotor-stimulating effects of cocaine.

\section{Striatal RGS7 Has an Essential Role in Controlling Cocaine Locomotor Sensitization}

As RGS9-deficient mice show increased cocaine sensitivity (Rahman et al, 2003), the lack of the effect of R7BP ablation observed in this study is unexpected because the levels of RGS9 in striatum of $\mathrm{R}^{\mathrm{B}} \mathrm{BP}^{-1-}$ mice are severely reduced and mice show similar coordination deficits in rotarod test. However, in addition to RGS9-2, striatal R7BP also binds to RGS7 and the abundance of RGS7-R7BP complexes is determined by RGS9-2 concentration (Anderson et al, 2009a). This prompted us to evaluate the contribution of striatal RGS7 to determining the sensitivity of cocaine action on locomotor activity. Our strategy was to analyze behavioral responses of mice with selective reduction in RGS7 expression in the striatum. We first screened for synthetic micro-RNA constructs identified sequences that could effectively knockdown RGS7 expression in transfected cell lines (Supplementary Figure S2a), and the construct that showed the highest efficiency (miR-427) was used to generate a recombinant lentivirus. On infection of primary striatal neurons with $4 \times 10^{9}$ particles per milliliter, the
miR-427 lentivirus reduced the levels of RGS7 by approximately twofold (Supplementary Figure S2b). As with the lentiviral rescue of R7BP, effectiveness of RGS7 knockdown virus was also tested at the in vivo level (Figure $6 \mathrm{a}$ and $\mathrm{b}$ ). Given the large volume of the dorsal striatum and the expected confinement of knockdown effectiveness limited to the population of neurons around the site of injection, test experiments were performed with a larger amount of the viruses than normally used for the behavioral experiments ( $\sim$ sixfold increase). Furthermore, for the subsequent biochemical analysis, sections located only around the plane of viral injections were selected. As shown in Figure 6a, unilateral injection of test mice with lentivirus targeting RGS7 resulted in approximately 35\% downregulation of RGS7 as compared with its expression in the contra-lateral side that received control virus (Figure 6a). Importantly, knockdown of RGS7 did not affect the expression of RGS92, R7BP (Figure 6a), or their complex formation as evidenced by co-IP experiments (Figure 6b). It is likely that the biochemical measures reflect an aggregate change in RGS7 concentration in the region. On the basis of the immunohistochemical data of the lentiviral-mediated expression of R7BP, (Figure 3c) we expect uneven changes in RGS7 concentration across the striatum in which areas of high knockdown efficiency are intermingled with areas of intact RGS7 expression. Therefore, behavioral alterations caused by this genetic manipulation are likely to stem from the effects on the select striatal circuitries rather than global change in the function of the entire striatum.

When injected bilaterally into mouse striatum, virus targeting RGS7, but not control virus, led to a dramatic increase in the sensitivity of the mice to the locomotorsensitizing action of cocaine (Figure $6 \mathrm{c}$ ). At the same time, no difference in the baseline (saline-injected) locomotion was recorded between the animal groups. A two-way ANOVA analysis revealed a significant effect of trial $\left(\mathrm{F}_{(7,128)}=13.48, p<0.001\right)$ as both groups showed higher locomotor activity with each additional cocaine injection (ie, sensitization) and a significant effect of genotype $\left(\mathrm{F}_{(1,125)}=28.67, p<0.001\right)$. Evaluation of mice in the rotarod task revealed that knockdown of RGS7 did not negatively affect motor learning behavior (Figure 6d). The mean latency \pm SEM to fall off the rotarod across all trials were $175 \pm 6.5$ (Control KD) and $194 \pm 5.8$ (RGS7 KD). Overall, these results suggest that RGS7 expression level in the striatum substantially contribute to determining the sensitivity of the reinforcing effects of cocaine.

\section{DISCUSSION}

In this study, we describe an underlining complexity governing the regulation of dopamine-mediated signaling in the striatum by macromolecular complexes of two members of $G$ protein signaling regulators: RGS7 and RGS9-2. Previous studies have established an essential role of RGS9-2 as a negative regulator of D2 and $\mu$-opioid receptor function by showing that its elimination in mice leads to: (i) enhanced locomotor activity and reward behavior in response to drugs such as cocaine (Rahman et al, 2003; Kovoor et al, 2005), and morphine (Zachariou et al, 2003; Psifogeorgou et al, 2007), (ii) motor 
a
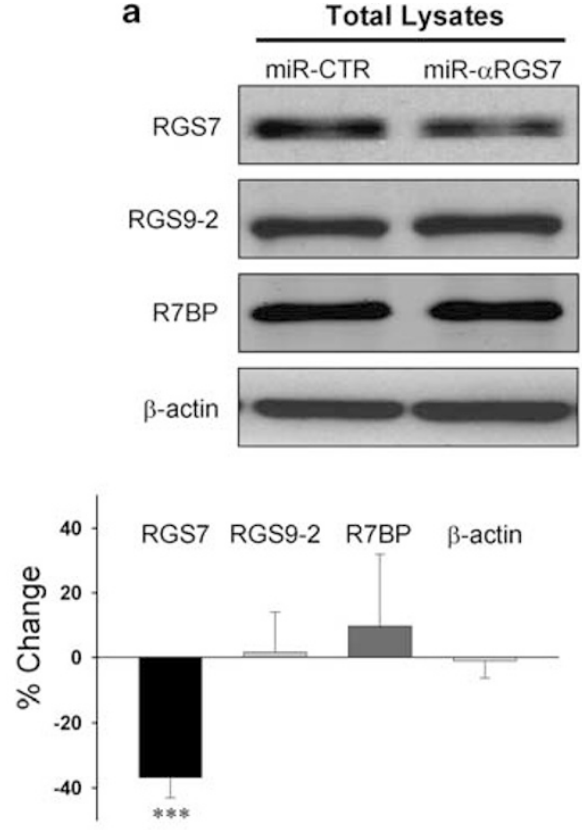

C

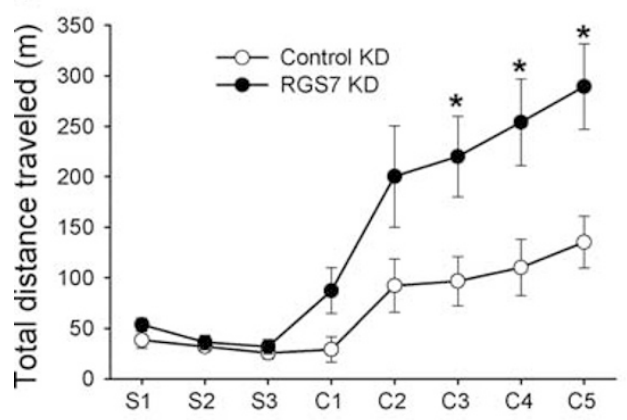

b

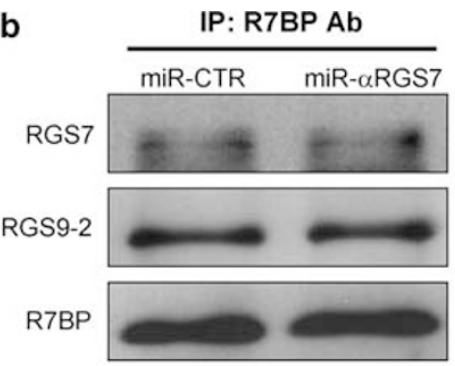

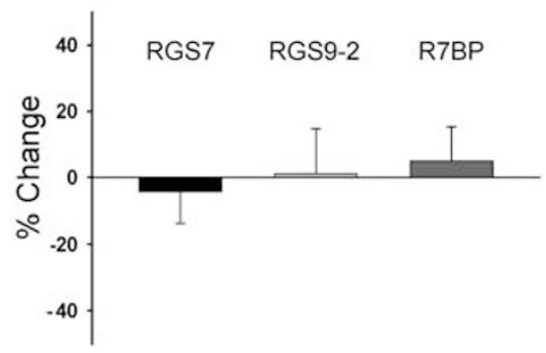

d

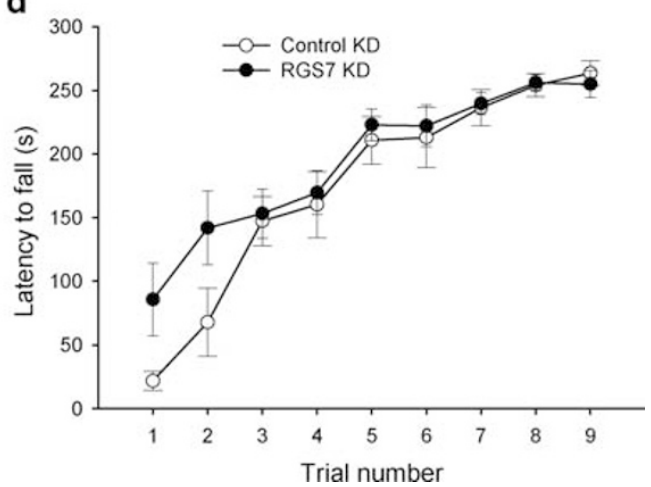

Figure 6 Knockdown of RGS7 in the striatum potentiates locomotor sensitization to cocaine. (a) Verification of lentivirus-mediated RGS7 knockdown in wild-type mice. Wild-type C57BL/6) mice at postnatal day 5 were unilaterally injected (600 nl per injection site) with either miR-CTR control knockdown lentivirus or miR- $\alpha$ RGS7 knockdown lentivirus into the striatum similarly as illustrated in Figure 3a. At postnatal day 25, brains were sectioned at 300- $\mu$ m thickness, and miR- $\alpha$ RGS7/miR-CTR lentivirus injected striatum was isolated. Tissue sections were solubilized with I\% Triton X-I00 containing IP buffer, $10 \mu \mathrm{g}$ of total extracted protein was separated on 12.5\% PAGE, and subjected to western blot analysis with the indicated antibodies. Shown is a representative maximal effect of RGS7 knockdown from one 300- $\mu$ m section. Quantification of protein change shown below was performed from three maximal RGS7 knockdown sections per brain, with two mice analyzed. Image analysis was performed by densitometry quantification using Image software package. Analysis of the data by Student's $t$-test, revealed a statistically significant difference between relative changes in RGS7 protein compared with $\beta$-actin (**** $p<0.00 \mathrm{I}$ ), and no difference $(p>>0.05)$ in relative changes between RGS9-2 and R7BP protein compared with $\beta$-actin. (b) Western blot analysis of proteins IP by $2 \mu \mathrm{g}$ of anti-R7BP antibody from the total lysates shown in panel (a). Densitometry quantification of proteins present in immunoprecipitated fractions are presented below. (c) Knockdown of RGS7 potentiates sensitization to cocaine. Wild-type mice were bilaterally injected with either RGS7 knockdown (RGS7 KD) lentivirus $(n=10)$ or control knockdown (Control KD) lentivirus $(n=8)$ with 90 nl per injections site. At 8 weeks of age, animals were given daily injections of saline for 3 days (SI-3), followed by five daily injections of $10 \mathrm{mg} / \mathrm{kg}$ cocaine (CI-5). A two-way ANOVA (day by genotype) revealed a significant effect of day $\left(F_{(7,128)}=13.48, p<0.001\right)$ as both groups showed higher locomotor activity with each additional cocaine injection (ie, sensitization). Injection of RGS7 KD virus produced higher responses compared with the injection with control virus $\left(F_{(1,125)}=28.67, p<0.00 \mathrm{I}\right)$ and a higher degree of sensitization as indicated by a statistically significant interaction between day and virus $\left(F_{(7,128)}=2.42\right.$, $\left.p=0.023\right)$. (d) RGS7 knockdown does not hamper motor learning performance as tested by rotarod test. A two-way ANOVA (trial by genotype) followed by Tukey's test revealed a significant improvement in rotarod performance with RGS7 knockdown virus within the first two trials $(p<0.05)$, but no significant difference between the two viruses for the remaining trials.

coordination/learning deficits (Blundell et al, 2008), and (iii) an increased susceptibility to antipsychotic- (Kovoor et al, 2005) and L-DOPA- (Gold et al, 2007) induced tardive dyskinesia. Recent studies suggest that RGS9-2 does not function by itself but rather acts as a part of a macromolecular complex with its two subunits: $\mathrm{G} \beta 5$ and membrane anchor R7BP (see Anderson et al, 2009b;
Jayaraman et al, 2009; Traynor et al, 2009 for recent reviews). Association with both G $\beta 5$ and R7BP is important for controlling proteolytic stability of RGS9-2 (Chen et al, 2003; Anderson et al, 2007b) and hence determines its expression levels in the striatum (Anderson et al, 2007b). Binding to R7BP is also required for targeting of RGS9-2 to plasma membrane and postsynaptic density compartments 
of striatal neurons (Anderson et al, 2007b). In striatal neurons, R7BP is associated not only with RGS9-2 but also with a second RGS protein, RGS7. Furthermore, the extent of RGS7-R7BP coupling was found to be dictated by RGS9-2 concentration as both RGS proteins compete for R7BP binding (Anderson et al, 2009a).

By characterizing $\mathrm{R} \mathrm{BP}^{-1-}$ mice in this study, we sought to establish the physiological role of $\mathrm{R} 7 \mathrm{BP}$ and its constituent RGS complexes in regulation of behaviors controlled by striatal signaling. We used behavioral paradigms of motor coordination/learning and locomotor responses to opiate and dopamine receptor stimulation that have proven to be useful for delineating RGS9-2 functions in the past. We found that mice lacking R7BP show deficits in motor coordination similar to that previously reported for $\mathrm{RGS}^{-1-}$ mice (Blundell et al, 2008), consistent with the dramatic reduction in the levels of RGS9-2 observed on the elimination of R7BP (Anderson et al, 2007b). As lentivirusmediated restoration of R7BP specifically in the striatum was accompanied by partial rescue of RGS9-2 expression and led to a considerable improvement of mouse performance in rotarod task, we conclude that normal motor coordination requires concerted contributions of striatal RGS9-2 and R7BP.

Interestingly, we find that deficits in motor coordination of $\mathrm{R} \mathrm{BP}^{-1-}$ mice are not apparent on initial trials but become pronounced when animals undergo several training sessions. As cortico-striatal circuitry is becoming increasingly accepted as a substrate that mediates motor learning (Packard and Knowlton, 2002; Costa et al, 2004; Pisani et al, 2005), these observations suggest that RGS9-2/R7BP complexes have an important role in striatum-mediated motor learning. Similar, although more pronounced, deficits in striatal motor learning are observed on elimination of NMDA receptor R1 subunit specifically in RGS9-2 expressing neurons (Dang et al, 2006) and on perturbation of molecules known to modulate NMDAR function in the striatum such as Cdk5 (Meyer et al, 2008) and PKA (Brandon et al, 1998). Furthermore, motor coordination deficits are observed in several mouse models with altered dopamine signaling including knockout of the dopamine transporter (Fernagut et al, 2003), along with D1 (Short et al, 2006) and D2 (Fowler et al, 2002) receptor knockouts. Given that dopamine-mediated control of glutamate responses through NMDA receptors is altered in $\mathrm{RGS9}^{-1-}$ mice (Kovoor et al, 2005) and that RGS9-2 has been found to be localized in the postsynaptic densities of spines (Anderson et al, 2007b) in which it could physically interact with NMDA receptors via $\alpha$-actinin 2 adapter (Bouhamdan et al, 2006), it seems plausible to suggest that RGS9-2/R7BP complexes are involved in modulation of dopamine signaling to the NMDAR that controls motor learning in the striatum.

Evaluation of $\mathrm{R} \mathrm{BP}{ }^{-I-}$ mice for their locomotor responses to opioids and cocaine have revealed striking differences that help dissect the underlying complexity of $G$ protein signaling in the striatum. We found that although elimination of R7BP resulted in a predicted increase in the sensitivity of mice to the locomotor effects of morphine (Zachariou et al, 2003; Psifogeorgou et al, 2007), their responses to cocaine administration remained unaltered, contrary to what is expected based on the severe reduction in RGS9-2 levels in $\mathrm{R} \mathrm{BP}^{-1-}$ mice (Anderson et al, 2007b) and reports that RGS9-2 acts as a negative regulator of the D2 receptor signaling (Rahman et al, 2003). The insights provided by these observations are twofold. First, they suggest that the increased sensitivity to opioids and psychostimulants previously reported for $\mathrm{RGS9}^{-1-}$ mice likely involves simultaneous alteration of $\mu$-opioid and D2 dopamine receptor signaling regulation that appears to be differentially controlled by distinct R7BP-RGS complexes in the striatum. Second, they indicate that regulation of dopamine signaling sensitivity is dictated not merely by RGS9-2 concentrations but rather involves three-way balancing between RGS9-2, RGS7, and R7BP. Indeed, consistent with this idea, we observe that manipulation of RGS7 levels in the striatum leads to profound changes in the locomotor responses of mice to cocaine administration. We have recently reported that both RGS9-2 and RGS7 are equally abundant in the striatum, yet they are unequally coupled to R7BP (Anderson et al, 2009a). Although a high percentage of RGS9-2 $(\sim 80 \%)$ is coupled to R7BP, only $\sim 20 \%$ of RGS7 exists in such complexes. Further, we have found that a decrease in RGS9-2 concentration triggers remodeling of these complexes, which enhances RGS7 coupling to R7BP. The extreme case of such remodeling is observed in RGS9 ${ }^{-1-}$ mice in which in the absence of RGS92, most of the striatal RGS7 is bound to R7BP, leading to a substantial change in its subcellular localization. Therefore, enhanced cocaine sensitivity reported for $\mathrm{RGS}^{-1-}$ mice might involve alteration of RGS7 function and thus must be considered when interpreting molecular mechanisms underlying sensitivity to cocaine action.

How can an increase in RGS7-R7BP coupling and decrease in RGS7 levels both lead to the same outcome of increased locomotor stimulation by cocaine? Mechanistically, we think this can be explained by the fact that both RNAi manipulation and increased coupling to R7BP observed in $\mathrm{RGS9}^{-1-}$ mice leads to the reduction of free RGS7. At this time, the signaling pathways regulated by RGS7 in the striatum are unknown and it appears possible that alteration of its function could affect the sensitivity of psychostimulant action by modulating GPCRs distinct from the ones controlled by RGS9-2. Furthermore, the effects of R7BP on the ability of R7 RGS proteins to regulate GPCR signaling are far from being understood, and it is possible that R7BP might regulate RGS9-2 and RGS7 function very differently. It is equally possible that R7BP exerts differential effects on the same RGS protein depending on the specific GPCR involved. Indeed, R7BP has been shown to stimulate the activity of RGS7 on Gi/o-coupled muscarinic M2 receptor (Drenan et al, 2005, 2006) but was also found to inhibit the regulation of the Gq-coupled muscarinic M3 receptor (Narayanan et al, 2007). Finally, GAP activities of RGS9-2 and RGS7 differ in their potencies toward individual members of the Gi/o class of $\mathrm{G}$ proteins (Hooks et al, 2003), and changes in RGS protein recruitment to postsynaptic sites is expected to alter both the extent and selectivity of GPCR regulation in the region. Therefore, future studies will need to establish GPCR specificity of RGS7 and RGS9-2 action in the striatum as well as the contribution of R7BP in establishing this selectivity.

In summary, the results presented in this study for the first time implicates RGS7 in controlling the sensitivity of 
cocaine-induced locomotor sensitization, describes the role of R7BP in controlling striatal motor functions, and provides evidence implicating interactions between two RGS proteins in control of dopamine signaling in the striatum.

\section{ACKNOWLEDGEMENTS}

We thank Dr William Simonds (NIH/NIDDKD) for the generous gift of anti- RGS7 and R7BP antibodies. This work was supported by NIH grants DA021743 (KAM), DA026405 (KAM), F31 DA024944 (GRA), NS047399 (GJG), F31 NS047399 (SD), MH061933 (KW), DA011806 (KW), DA019666 (MJT), and a McKnight Land-Grant Award (KAM).

\section{DISCLOSURE}

The authors declare that except for the income received from the primary employers and NIH grants listed in the 'Acknowledgements' section below no financial compensation has been received from any individual or corporate entity over the past 3 years of research or professional service that could be perceived as constituting a potential conflict of interest.

\section{REFERENCES}

Anderson GR, Lujan R, Martemyanov KA (2009a). Changes in striatal signaling induce remodeling of RGS complexes containing Gbeta5 and R7BP subunits. Mol Cell Biol 29: 3033-3044.

Anderson GR, Lujan R, Semenov A, Pravetoni M, Posokhova EN, Song JH et al (2007b). Expression and localization of RGS9-2/ Gbeta5/R7BP complex in vivo is set by dynamic control of its constitutive degradation by cellular cysteine proteases. J Neurosci 27: 14117-14127.

Anderson GR, Posokhova E, Martemyanov KA (2009b). The R7 RGS protein family: multi-subunit regulators of neuronal $G$ protein signaling. Cell Biochem Biophys 54: 33-46.

Anderson GR, Semenov A, Song JH, Martemyanov KA (2007a). The membrane anchor R7BP controls the proteolytic stability of the striatal specific RGS protein, RGS9-2. J Biol Chem 282: 4772-4781.

Blundell J, Hoang CV, Potts B, Gold SJ, Powell CM (2008). Motor coordination deficits in mice lacking RGS9. Brain Res 1190: 78-85.

Bouhamdan M, Yan HD, Yan XH, Bannon MJ, Andrade R (2006). Brain-specific regulator of G-protein signaling 9-2 selectively interacts with alpha-actinin-2 to regulate calcium-dependent inactivation of NMDA receptors. J Neurosci 26: 2522-2530.

Brandon EP, Logue SF, Adams MR, Qi M, Sullivan SP, Matsumoto $\mathrm{AM}$ et al (1998). Defective motor behavior and neural gene expression in RIIbeta-protein kinase A mutant mice. J Neurosci 18: 3639-3649.

Burchett SA (2005). Psychostimulants, madness, memory, and RGS proteins? Neuromolecular Med 7: 101-127.

Cabrera-Vera TM, Hernandez S, Earls LR, Medkova M, SundgrenAndersson AK, Surmeier DJ et al (2004). RGS9-2 modulates D2 dopamine receptor-mediated $\mathrm{Ca} 2+$ channel inhibition in rat striatal cholinergic interneurons. Proc Natl Acad Sci USA 101: 16339-16344.

Chen CK, Eversole-Cire P, Zhang HK, Mancino V, Chen YJ, He W et al (2003). Instability of GGL domain-containing RGS proteins in mice lacking the $\mathrm{G}$ protein beta-subunit Gbeta5. Proc Natl Acad Sci USA 100: 6604-6609.
Coleman JE, Huentelman MJ, Kasparov S, Metcalfe BL, Paton JF, Katovich MJ et al (2003). Efficient large-scale production and concentration of HIV-1-based lentiviral vectors for use in vivo. Physiol Genomics 12: 221-228.

Costa RM, Cohen D, Nicolelis MA (2004). Differential corticostriatal plasticity during fast and slow motor skill learning in mice. Curr Biol 14: 1124-1134.

Dang MT, Yokoi F, Yin HH, Lovinger DM, Wang Y, Li Y (2006). Disrupted motor learning and long-term synaptic plasticity in mice lacking NMDAR1 in the striatum. Proc Natl Acad Sci USA 103: 15254-15259.

Davidson S, Truong H, Nakagawa Y, Giesler GJ (2010). A microinjection technique for targeting regions of embryonic and neonatal mouse brain in vivo. Brain Res 1307: 43-52.

DeLong MR, Wichmann T (2007). Circuits and circuit disorders of the basal ganglia. Arch Neurol 64: 20-24.

Drenan RM, Doupnik CA, Boyle MP, Muglia LJ, Huettner JE, Linder $\mathrm{ME}$ et al (2005). Palmitoylation regulates plasma membrane-nuclear shuttling of R7BP, a novel membrane anchor for the RGS7 family. J Cell Biol 169: 623-633.

Drenan RM, Doupnik CA, Jayaraman M, Buchwalter AL, Kaltenbronn KM, Huettner JE et al (2006). R7BP augments the function of RGS7/Gbeta5 complexes by a plasma membranetargeting mechanism. J Biol Chem 281: 28222-28231.

Fernagut PO, Chalon S, Diguet E, Guilloteau D, Tison F, Jaber M (2003). Motor behaviour deficits and their histopathological and functional correlates in the nigrostriatal system of dopamine transporter knockout mice. Neuroscience 116: 1123-1130.

Fowler SC, Zarcone TJ, Vorontsova E, Chen R (2002). Motor and associative deficits in D2 dopamine receptor knockout mice. Int J Dev Neurosci 20: 309-321.

Gainetdinov RR, Premont RT, Bohn LM, Lefkowitz RJ, Caron MG (2004). Desensitization of $G$ protein-coupled receptors and neuronal functions. Annu Rev Neurosci 27: 107-144.

Gold SJ, Hoang CV, Potts BW, Porras G, Pioli E, Kim KW et al (2007). RGS9-2 negatively modulates L-3,4-dihydroxyphenylalanine-induced dyskinesia in experimental Parkinson's disease. J Neurosci 27: 14338-14348.

Grabowska D, Jayaraman M, Kaltenbronn KM, Sandiford SL, Wang Q, Jenkins S et al (2008). Postnatal induction and localization of R7BP, a membrane-anchoring protein for regulator of $\mathrm{G}$ protein signaling 7 family-Gbeta5 complexes in brain. Neuroscience 151: 969-982.

Groenewegen HJ (2003). The basal ganglia and motor control. Neural Plast 10: 107-120.

Hollinger S, Hepler JR (2002). Cellular regulation of RGS proteins: modulators and integrators of $\mathrm{G}$ protein signaling. Pharmacol Rev 54: 527-559.

Hooks SB, Waldo GL, Corbitt J, Bodor ET, Krumins AM, Harden TK (2003). RGS6, RGS7, RGS9, and RGS11 stimulate GTPase activity of Gi family G-proteins with differential selectivity and maximal activity. J Biol Chem 278: 10087-10093.

Hyman SE, Malenka RC, Nestler EJ (2006). Neural mechanisms of addiction: the role of reward-related learning and memory. Annu Rev Neurosci 29: 565-598.

Jayaraman M, Zhou H, Jia L, Cain MD, Blumer KJ (2009). R9AP and R7BP: traffic cops for the RGS7 family in phototransduction and neuronal GPCR signaling. Trends Pharmacol Sci 30: $17-24$.

Kovoor A, Seyffarth P, Ebert J, Barghshoon S, Chen CK, Schwarz S et al (2005). D2 dopamine receptors colocalize regulator of G-protein signaling 9-2 (RGS9-2) via the RGS9 DEP domain, and RGS9 knock-out mice develop dyskinesias associated with dopamine pathways. J Neurosci 25: 2157-2165.

Kreitzer AC (2009). Physiology and pharmacology of striatal neurons. Annu Rev Neurosci 32: 127-147.

Kreitzer AC, Malenka RC (2008). Striatal plasticity and basal ganglia circuit function. Neuron 60: 543-554. 
Martemyanov KA, Yoo PJ, Skiba NP, Arshavsky VY (2005). R7BP, a novel neuronal protein interacting with RGS proteins of the R7 family. J Biol Chem 280: 5133-5136.

Meyer DA, Richer E, Benkovic SA, Hayashi K, Kansy JW, Hale CF et al (2008). Striatal dysregulation of $\mathrm{Cdk} 5$ alters locomotor responses to cocaine, motor learning, and dendritic morphology. Proc Natl Acad Sci USA 105: 18561-18566.

Narayanan V, Sandiford SL, Wang Q, Keren-Raifman T, Levay K, Slepak VZ (2007). Intramolecular interaction between the DEP domain of RGS7 and the Gbeta5 subunit. Biochemistry 46: $6859-6870$.

Nestler EJ (2005). Is there a common molecular pathway for addiction? Nat Neurosci 8: 1445-1449.

Nicola SM, Surmeier J, Malenka RC (2000). Dopaminergic modulation of neuronal excitability in the striatum and nucleus accumbens. Annu Rev Neurosci 23: 185-215.

Packard MG, Knowlton BJ (2002). Learning and memory functions of the Basal Ganglia. Annu Rev Neurosci 25: 563-593.

Paxinos G, Halliday G, Watson C, Koutcherov Y, Wang HQ (2007). Atlas of the Developing Mouse Brain. Elsevier: Amsterdam, The Netherlands.

Pierce RC, Kumaresan V (2006). The mesolimbic dopamine system: the final common pathway for the reinforcing effect of drugs of abuse? Neurosci Biobehav Rev 30: 215-238.
Pisani A, Centonze D, Bernardi G, Calabresi P (2005). Striatal synaptic plasticity: implications for motor learning and Parkinson's disease. Mov Disord 20: 395-402.

Pravetoni M, Wickman K (2008). Behavioral characterization of mice lacking GIRK/Kir3 channel subunits. Genes Brain Behav 7: 523-531.

Psifogeorgou K, Papakosta P, Russo SJ, Neve RL, Kardassis D, Gold SJ et al (2007). RGS9-2 is a negative modulator of muopioid receptor function. J Neurochem 103: 617-625.

Rahman Z, Schwarz J, Gold SJ, Zachariou V, Wein MN, Choi KH et al (2003). RGS9 modulates dopamine signaling in the basal ganglia. Neuron 38: 941-952.

Short JL, Ledent C, Drago J, Lawrence AJ (2006). Receptor crosstalk: characterization of mice deficient in dopamine D1 and adenosine A2A receptors. Neuropsychopharmacology 31: 525-534.

Sondek J, Siderovski DP (2001). Ggamma-like (GGL) domains: new frontiers in G-protein signaling and beta-propeller scaffolding. Biochem Pharmacol 61: 1329-1337.

Traynor JR, Terzi D, Caldarone BJ, Zachariou V (2009). RGS9-2: probing an intracellular modulator of behavior as a drug target. Trends Pharmacol Sci 30: 105-111.

Zachariou V, Georgescu D, Sanchez N, Rahman Z, DiLeone R, Berton O et al (2003). Essential role for RGS9 in opiate action. Proc Natl Acad Sci USA 100: 13656-13661.

Supplementary Information accompanies the paper on the Neuropsychopharmacology website (http://www.nature.com/npp) 